Doi: $10.24234 / \mathrm{se} .2020 .2 .2 .232$

\title{
HIGHLIGHTS OF PRINCIPLES USED FOR AUGMENTATIVE AND ALTERNATIVE COMMUNICATION ASSESSMENT IN PATIENTS WITH CENTRAL NERVOUS SYSTEM DISORDERS
}

\author{
AUTHOR'S DATA: \\ Hasmik Mkrtchyan, MSc, Assistant professor \\ Department of Kinesiology, Armenian State Institute of Physical Culture and Sports, Armenia \\ University lecturer \\ Contacts: hasmikmh@mail.ru
}

\begin{abstract}
Different technologies are used, including Augmentative and Alternative Communication (AAC), which can improve communication competences and life participation in patients with neurological conditions and communication deficits. The assessment of patients for the AAC need is a complex procedure encompassing not only speech and language assessment but also evaluation of cognitive, motor, visual, auditory, perceptive, and a variety of other general factors that influence the process of AAC method selection. Different standardized linguistic tools are used in the assessment process. Reassessments are an indisputable part of the AAC intervention process. Training of patients, caregivers, and other primary communication partners is imperative to ensure the successful use of communication technologies. The patients must take part in assessor controlled device trials to reveal the most appropriate AAC technology. Whenever needed, the AAC training ensures the permanent use of the technologies, providing ongoing support to the families once the skilled intervention is discontinued.
\end{abstract}

Keywords: Augmentative and Alternative Communication, assessment, intervention, neurological conditions, training, assessment tools.

\section{INTRODUCTION}

The process of decision-making for augmentative and alternative communication (AAC) assessment is based on a range of strategies and technologies that apply a variety of modalities, 
including no technology (no tech), low technology (low tech), and high technology (high tech) methods. The types of AAC methods that are effectively used in practice for patients with neurological conditions (cerebral palsy, traumatic brain injury, stroke, and other syndromes) are usually selected depending on the severity and size of the brain lesion and on the stage of recovery/rehabilitation process that patients are involved. In the majority of cases, it is a common practice for AAC intervention to be initiated with delay, and speech therapists usually apply AAC strategies only after the patient's recovery and associated communication disorders are stabilized (Fager, 2005). Different models of AAC intervention that are currently used in practice require methods which change and develop throughout the rehabilitation process to meet the altered/improved needs of the person, particularly for people with communication deficit related to neurological conditions (Fager, Doyle \& Karantounis, 2007; Campbell, Baladin, \& Togher, 2002; Deruyter \& Donaghue, 1989). The primary goal of the AAC intervention is to facilitate a patient's ability to express their basic needs, to ensure their participation in decision-making about their care, and actively take part in therapy. Therefore, the AAC interventions ideally are initiated in the acute stages of rehabilitation. The speech therapists are forced to changes the AAC methods used in the course of recovery. These changes are anticipated throughout the rehabilitation program, and the expectation of change affects the decision of specialists about modes of interventions selected at different stages of recovery. Based on these approaches, the temporary use of no-tech strategies and low tech methods are usually applied in the initial phase of recovery, especially when the therapist can predict the positive outcome and register ongoing improvements. This strategy envisages the gradual improvement of AAC technology being used and the selection of high tech systems. As a rule, the high tech technologies are costlier, require a much longer period for training, and greater cognitive capacity is needed to control them effectively.

The development or change of the AAC device/method should be conducted with observations concentrated on the changing needs of the patient. In the early stages of neurorehabilitation, the patients with neurological deficit associated with altered communication may need the use of an AAC method, but the selection of the AAC strategy is influenced by a variety of factors, such as changes in the degree of alertness, the stability of general medical condition, type of pharmacotherapy, secondary complications, pain syndrome, and endurance. The AAC intervention usually applies the strategy of multimodal stimulation based on the involvement of different verbal and nonverbal tools to improve communicative competencies and intervention 
efficacy. In the majority of neurological patients' effectiveness of AAC use aiming to support communication skills required to satisfy the basic needs of a person using the simplest response categories (the maximum shortest response, e.g. yes/no), could be achieved by applying simple, low tech strategies (picture exchange, symbols or alphabet boards) (Light, 1988). In the second or middle stage of rehabilitation, the patients are adapted and more skilled in the use of low tech interventions, which depends on the time they have spent with the AAC method and experience/training of the communication partner. The second phase of neurorehabilitation has certain specific determinants. Patients with neurological deficits have improved cognitive skills, improved or recovered memory, and a higher level of alertness. All these factors contribute to the improvement in communicative skills. The individuals, therefore, can use more improved forms of alternative communication. In the last third stage of neurorehabilitation, the patients are less dependent on communication partners and possess better motor and communication skills to use high tech strategies. The third or late stage is conducted not in clinical settings, and the patients can receive AAC services in different community settings (support centers, offices of a speech therapist, outpatient clinics).

The selection of the AAC method greatly depends on the type of the neurological condition, and the type of communication disorder associated with the neurological condition. Patients with aphasia may experience a whole range of specific problems in using AAC strategies when compared to individuals with a different type of motor speech problems. Individuals with aphasia have limited ability to independently select and if necessary combine AAC methods and to control the selected communication systems. This limited ability is due to different degrees of the linguistic deficit. When selecting an AAC mode for this category of patients, who are considered partner-dependent because of aphasia, special training and instructions are needed for the patient and the communication partner (Lasker, Garrett \& Fox, 2007). The speech therapist has to provide the patient with aphasia with a range of AAC methods and to help and support the patient in the selection process. The therapist has to ensure that proper selection is made which can facilitate and improve the communication skills of the patient and help him to become a less dependent communicator (Garrett \& Lasker, 2005). 


\section{BACKGROUND FOR THE ASSESSMENT PROCESS}

The communication process integrates different functional competencies: physical, mental, emotional, and behavioral. The interaction of these abilities provides proper communication skills. Assessment and interventional strategies in patients with communication disorders require a team approach, involving specialists who are competent enough to assess and restore the altered function. The AAC team must include not only the speech therapist, AAC specialist, ergo therapists, physical therapist, psychologist, and special educator, but also the caregivers. When planning for the assessment process the team members have to focus on the peculiarities of communication deficit, and priorities mentioned by the family members or caregivers of the individual with communicative dysfunction.

Patients with congenital or acquired neurological conditions may manifest different communication problems. A category of patients with cortical lesions in the brain may have aphasia when they lose the ability to use the language or understand it. Aphasia leads to receptive and expressive speech dysfunction, including the loss of not only verbal skills. Writing, typing, reading skills are lost together with speech and comprehension. The category of patients with neurological and communication deficit experiences also problems in social communication when trying to use pragmatic language skills (eye contact, turn-taking, initiation of conversation, etc.). The main type of motor dysfunction is usually dysarthria when speech disturbance is due to changes in articulatory muscle strength and tone. Speech apraxia is manifested by the increased level of efforts to produce speech, and lack of coordination in articulatory muscle contractions and oral movements, vocal changes (quality of voice and pitch) induced by not proper breath support, and dysfluency. Comprehension of patients is affected also by the lack of cognitive skills (Tobii, 2010).

Different linguistic competence measurement tools are used to evaluate the communication abilities of patients when considering different AAC options (Kertesz, et al 2007). The linguistic competence assessment tools (Kertesz, 2007; Dunn \& Dunn, 2007; Semel, Wiig \& Secord, 2003) and their brief descriptions are presented in table 1 . 
Table 1.

Tools used for the linguistic assessment

\begin{tabular}{|l|l|}
\hline Western Aphasia Battery Revised & $\begin{array}{l}\text { The Western Aphasia Battery was designed to provide the } \\
\text { means of evaluating major clinical aspects of language } \\
\text { function: content, fluency, auditory comprehension, } \\
\text { repetition, and naming plus reading, writing, and } \\
\text { calculation (Kertesz, 2007). }\end{array}$ \\
\hline $\begin{array}{l}\text { Clinical Evaluation of Language } \\
\text { Fundamentals -Fourth Edition }\end{array}$ & $\begin{array}{l}\text { CELF-IV is used to identify, diagnose, and perform a } \\
\text { follow-up evaluation of language skill deficits in children, } \\
\text { adolescents, and young adults (from 6 years to 21 years). It } \\
\text { was designed to identify individuals lacking the basic } \\
\text { foundations of content and form that characterize the } \\
\text { language use: word meanings (semantics), word and } \\
\text { sentence structure (morphology and syntax), as well as the } \\
\text { recall and retrieval of spoken language (memory) (Semel, } \\
\text { Wiig \& Secord, 2003). }\end{array}$ \\
\hline $\begin{array}{l}\text { Peabody Picture Vocabulary } \\
\text { Test, Fourth Edition }\end{array}$ & $\begin{array}{l}\text { (PPVT-R) measures an individual's receptive vocabulary } \\
\text { and provides, at the same time, a quick estimate of verbal } \\
\text { ability or scholastic aptitude (Dunn \& Dunn, 2007). }\end{array}$ \\
\hline
\end{tabular}

The linguistic assessment helps to gather information about a patient's level of letter/symbol recognition (e.g. the patient tries to match pictures to words, etc.) and their written skills when trying to type answers to questions. Such an assessment contributes to the decisionmaking, helps to determine the symbols and language structure a patient might be able to use with selected AAC methods. Other than linguistic data the assessor can gather information from not structured observations studying the ways a patient communicates with partners and family members, as well as the strategies used by the patients, in cases when they are trying to augment speech verbalization using gestures or explicit facial expression.

The above-discussed tests are not able to determine whether a patient is appropriate for the AAC, or what type of AAC could be effective, but they explicitly reveal the areas or domains that should be targeted and a thorough analysis of the patient's weaknesses is a strong predicting factor in determining what type of AAC may provide with successful compensation.

A somewhat different approach is necessary when assessing children with communication deficits. In this stratum of patients, the assessment process requires a developmental approach when not only the language competencies of the patient are assessed, but also the stage of development. In older children and adolescents an ecological approach is used instead of the 
developmental assessment method. The ecological approach assesses the different functions of the patient concerning the environment and activities in which the patient participates. The ecological method and tools applied for this assessment method were developed by Brown et al in 1979. These tools are a helpful measure guiding the analysis of the environment and meaningful activities a patient is involved in (Brown. et al 1979). The difference between ecological and functional communication assessment is that the later includes standardized protocols such as the Communication Activities of Daily Living (Holland, Frattali \& Fromm, 1999), or questionnaires for caregivers - ASHA FACS (Frattali, Thompson, Holland, Wohl \& Ferketic, 1995). The functional assessment tools are useful when assessing adult patients to reveal a regularity of how a patient's communication deficit affects their functions and participation in real-life activities. Special efforts and time is required to reveal the patient's values and priorities; and by targeting them facilitate the selective integration or targeting of outcomes that are essential and are considered as a priority for the patient and caregivers. Special assessment tools like the Communication Needs Assessment (Fager, Doyle \& Karantounis, 2007) or The Aphasia Needs Assessment (Garrett \& Lasker, 2005) are proper methods to obtain information about the patient's communication skills and competences which are used in settings where the patient communicates most often and can reveal the priorities in communication methods (e.g., email, reading, writing, etc.).

The therapist must include the patient with caregivers in the assessment process, in cases when the patient is not an adult. The involvement of the patient is very important, as this promotes their self-determination and acceptance of the selected AAC method. On the other hand, the caregivers (who usually are individuals who use the AAC with the patient) can provide information about settings in which functional communication is most needed and can influence the acceptance of the AAC method. Another category of people that can assist the therapist in the assessment process is the medical staff or patient's care providers. These people from the patient's environment can supplement information about the communication needs of the patient, as these individuals are taking part in daily functions with the patient and caregivers.

All these issues discussed do not complete the scope of factors that impact the communication of neurological patients and their ability to use AAC technologies. This scope includes also the cognitive functions (e.g. attention, memory, inhibitory control, etc.) that can directly influence the communicative competences of patients and may also impact the ability to 
use AAC strategies. According to previously published research reports (Rowland \& Schweigert, 2003), there are a variety of aspects related to cognition that can predetermine the patient's ability to use AAC methods. These aspects include memory, ability to understand relationships between people and objects, understanding of concrete and abstract symbolic representation, communicative intent, awareness, and metacognitive learning strategies. For the assessment process, the information representing the cognitive functions of patients is collected using neuropsychological assessment tools. The cognitive assessment is not always possible as the patients have speech or language impairment. The researchers and assessors are forced to use observational and descriptive data presenting the patient's performance during functional tasks or their test-taking behaviors. Multiple examples could be given describing the observational information (e.g., observations about initiation, attempts to make corrections, and patterns of visual search).

The use of different AAC methods, both the low and high tech methods, requires a certain level of motor skills. Motor skills are directly related to speech and some of them take part or influence the articulatory process. Motor skill assessment is required in patients with dysarthria, speech apraxia, and voice problems. These assessments are important to estimate the functional intelligibility of the patient, tested by various methods (cell phone, iPad, etc.) and in various settings (at school, at home, etc.). The use of AAC extremely depends on the use of an upper extremity. Different associated neurological conditions (paralysis, paresis, motor apraxia in patients with cerebral palsy, brain injury, or other conditions) hamper the person's ability to use a wide range of AAC tools. The input or access method in AAC technologies greatly depends on the upper extremity function (for example, to press a switch). Patients with apraxia who manifest with impaired motor planning and disturbed coordination may be confused when they have to select the target switch or push alternately several other switches. AAC specialists have to consider the medication that patients use to treat spasticity. Drug therapy may impact motor control and lead to reduced cognitive alertness, and/or articulatory musculature weakness. The reduced speech intelligibility is in some cases due to the side effects of pharmacotherapy. The mobility status of patients can affect the AAC method selection as well. A person who is walking needs a lighter device (e.g. Speech Generating Device (SGD), and those patients who are in a wheelchair can use heavier devices fixed to the wheelchair. 
Auditory function and vision may influence the capacity to use different AAC methods, as a majority of high and even low tech methods rely on sight for symbols and words. However, neurological patients with severely impaired vision can use some AAC technologies. Information about a patient's perceptual skills is essential in the assessment process, assisting the therapist with the method or device selection. Visual and tactile perception generates feedback for the AAC input when the patients have an auditory deficit.

There are other general factors, which have the potential to affect the ability of patients to use AAC. Medical instability, issues with behavior, decreased endurance, perceptual hypersensitivity, lack of financial resources, and psychological problems related to the acceptance of the acquired disability or need for AAC use.

A well-designed intervention plan is based entirely on a properly conducted assessment process. The assessment process of all functions should be followed by assessor-initiated devicetrials, trying to determine the most appropriate device/method. The intervention plan must include reassessment sessions to evaluate the continued success of the selected AAC method and to reveal additional communication needs of the patient. Reassessments are a necessary part of intervention strategy as the patient's communication recovers and the social environment continuously changes within the intervention process.

\section{CONCLUSIONS}

The assessment of patients for the AAC need is a complex procedure encompassing not only speech and language assessment but also evaluation of cognitive, motor, visual, auditory, perceptive, and a variety of other general factors that influence the process of AAC method selection. Reassessments are an indisputable part of the AAC intervention process.

Training of patients, caregivers, and other primary communication partners is imperative to ensure the successful use of communication technologies. The patients must take part in assessor controlled device trials to reveal the most appropriate AAC technology. Training of patients and communication partners must include the elaboration of prompts, selecting cues that require the least amount of effort. The prompts are essential when a patient is in pain or has other exacerbated conditions. Whenever needed AAC training ensures the permanent use of the technologies, providing ongoing support to the families once the skilled intervention is discontinued. 


\section{REFERENCE LIST}

1. Holland, A., Frattali, C., \& Fromm, D., (1999). Communication Activities (Daily Living (2nd Ed.), Pro-Ed, Austin, Texas.

2. Rowland, C., \& Schweigert, P.D. (2003). Cognitive Skills, Communicative Competence for individuals who use AAC, J. Light, D.R. Beukelman and 1. Reichle, eds, Paul H. Brooks Publishing Company, Baltimore. Maryland, pp. 241

3. Frattali, C., M., Thompson, C., K., Holland, A., L., Wohl, C., B., \& Ferketic, M., M. (1995). Functional Assessment (Communicat kville, Maryland.

4. Semel, E., Wiig, H., \& Secord, W. (2003ion Skills (Jr Adults (ASHA FACS), American Speech-Language Hearing Association, Roc). Clinical Evaluation of Language Fundamentals -Fourth Edition. The Psychological Corporation, San Antonio, Texas.

5. Deruyter, F., \& Donaghue, K.A. (1989). Communication and traumatic brain injury: A case study, Augmetltative and Alternative Communication 5, 49-54.

6. Light, J. (1988). Interaction involving individuals using augmentative communication systems: State of the art and future directions for research, Auwnellfative and AlTernative Communication 4. 66-82.

7. Lasker, J., P., Garrett, K., L, and Fox, L., E. (2007). Severe Aphasia in: Alternative Communication Strategies.for Adults with Acute or Chronic Medical conditions. D. Beukelman. K. Garrett and K. Yorkston, eds, Paul H. Brooks Publishing Company, Baltimore, Maryland, 2007, pp. 163-206.

8. Garrett, K., L., \& Lasker, J., P. (2005) Adults with severe aphasia. in: Augmentative and Alternative Communication: Supporting Children and AdulTS with Complex CommunicaTion Needs, D. Beukelman and P. Mirenda, eds, Paul H. Brooks Publishing Company, Baltimore, Maryland, pp. 467-516.

9. Garrett, K., L., \& Lasker, J., P. (2005). Adults with severe aphasia. in: Augmentative and Alternative Communication: Supporting Children and Adults with Complex Communication Needs, D. Beukelman and P. Mirenda, eds, Paul H. Brooks Publishing Company, Baltimore, Maryland, pp. 467-516.

10. Kertesz, A. (2007). Western Aphasia Battery Revised. Harcourt Assessment, San Antonio. Texas. 
11. Brown, L., Branson-McLean, M.B., Baumgart, D., Vincent, L., Falvey, M. \& Schroder, I. (1979). Using the characteristics of current and subsequent least restrictive environments in the development of curricular content for severely handicapped students, AAESPH Review 4,407-424.

12. Campbell, L., Baladin, S., \& Togher, L. (2002). Augmentative and Alternative Communication Use by People with Traumatic Brain Injury: A Review. Advances in Speech Language Pathology, 4(2), 89-94.

13. Dunn, L., M., \& Dunn, D., M. (2007). Peabody Picture Vocabulary Test, Fourth Edition, The Psychological Corporation, San Antonio. Texas.

14. Fager, S. (2005). Individuals with Traumatic Brain Injury, in: Alternative Communication: Supporting children and Adults wilh Complex Communication Needs. D.R. Beukelman and P. Mirenda, eds, Paul H. Brooks Publishing Company, Baltimore: Maryland, pp. 131162.

15. Fager, S., Doyle, M. \& Karantounis, R. (2007). Communication Needs Assessment, in: Augmentative Communicatio/l Strategies for Adults with Acute or Chrollic Medical Conditions. Beukelman, K.L. Garrett and K.M. Yorkston, eds, Paul Brookes Publishing Company, Baltimore. Maryland, pp. Worksheet 5.6.

16. Symbol Mate by Tobii, (2010). retrieved from http://www. tobiiati.com!corporate/ productslsymbolmate.aspx on June 25, 2010. 